\title{
Fabrication and properties of silver nanowires (AgNWs) functionalized fabric
}

\author{
Rony Mia ${ }^{1,2} \mathbb{D} \cdot$ Suraiya Sultana ${ }^{3}$
}

Received: 31 August 2020 / Accepted: 5 November 2020 / Published online: 22 November 2020

(c) Springer Nature Switzerland AG 2020

\begin{abstract}
The tremendous clarification of Fabric-based solicitation structures is hugely suggested for clothing hardware. The nanowire affiliations in which have high conductivity, low resistivity, high adaptability similarly as straightforwardness are developing in the field of electronic completes. The material of knitted fabric surface quickly utilized for passing on a substitute sort of adaptable then extraordinary electro sensor contraptions. Nylon/PU surfaces with silver nanowire (AgNW) and polydimethylsiloxane (PDMS) direct conductive flicks have been proposed as a promising likelihood to supplant particular silver shade of electronic conductivity establishment. This sort of nanostructure has high capacitance and wide electrochemical shallow other than an ideal particle dispersing course in the composed nanowire structure which is wound up being a perfect anode material for world-class supercapacitors. These models of supercapacitors use the high adaptability mind-blowing processability and broad capacitance of organizing polymers which attainably extend the procedure of supercapacitors. In this examination, we utilized a key Dip and Dry framework to make flexible pushing recognizing fabric surface from side to side the layer of the AgNW. So versatile nylon/PU surface consistency toward displaying electrical conductivity, moreover, make a polymer cathode by covering through the optically clear PDMS slim film. The AgNWs verified superficial delineated by the scanning electron microscopy (SEM) then the electromechanical complete besides strain to distinguish highlight of the AgNWs investigated. The mechanical properties of the surfaces did not change amazingly subsequently the conduct. The promising hydrophobic character of PDMS makes the sample super hydrophobic through the water disallowed and a dynamic establishment in wearable bits of outfit with electronic implements.
\end{abstract}

Keywords Silver nanowire · PDMS film · Electrical conductivity · Flexible strain sensor · Temperature · Human motion · Hydrophobicity

\section{Introduction}

Silver nanoparticle (AgNP) covers on materials have pulled in a lot of thought since AgNPs allow emerge material items, for instance, antibacterial improvement [1, 2] ultraviolet UV affirmation [3] conductivity [4] hydrophobicity [5] compound perseverance photosensitivity optical and electrical properties and reactant movement [6-8]. silver-based nanomaterials for example since AgNPs, silver nanowires (AgNWs) and silver cross segments ag structures have decimated in monstrous idea inferable from the most raised electrical and warm conductivity of silver among all materials and have arranged presentations in making nanoscale electronic devices [9-12].

AgNWs have been acquainted with different polymeric materials to make dainty direct movies. These polymers

Rony Mia, mroni_mia@yahoo.com; mroni_mia@niter.edu.bd | ${ }^{1}$ College of Chemistry and Chemical Engineering, Wuhan Textile University, Wuhan 430073, China. ${ }^{2}$ Department of Textile Engineering (Wet Processing), National Institute of Textile Engineering \& Research (NITER), Savar, Dhaka 1350, Bangladesh. ${ }^{3}$ Wuhan Textile University, State Key Laboratory of New Textile Materials and Advanced Processing Technologies, Ministry of Education, Wuhan 430073, China. 
have been manhandled as structures or cautious layers. Models are conductive polymers like PEDOT: PSS [13, 14] polycarbonate pc [15] and poly-acrylate father [16] or changed polymers like pet polydimethylsiloxane (PDMS), PVA, put PVDF [17-19]. The essential for adaptable lightweight electrically conductive contraptions actuated the time of watchful materials called conductive polymer nanocomposites CPC [20]. among all these conductive nanoparticles which have been comprehensively considered by specialist's AgNWs could achieve electrical conductivity flexibility and straightforwardness all in the meantime because of silver has the most raised electrical conductivity $6.3 \times 10^{7} \mathrm{~s} / \mathrm{m}$ among the majority of the metals and the likelihood of merging 1-d nanowires that could be adaptable and clear [21, 22]. In association with silver nanoparticle (AgNP) the exceedingly anisotropic AgNWs have the central focuses in keeping an infiltrated structure when manufactured as a film or connected with a cross section [23].

The interconnected nanowires film in a system will permit consistent passage of silver particles after the nanowires crumbling [24]. Likewise, AgNWs are known to be okay for people and widely utilized in adaptable gear [25-27]. In persistent an over the top time length; the critical idea has been paid to flexible criticalness gathering contraptions. The most essential section for collecting flexible supercapacitors is the acquisition of fitting adaptable terminals with high capacitance and high electrical conductivity to guarantee rapid charge-release process. Up to now, different sorts of current specialists are utilized in the course of action and creation of supercapacitors including metal foils $[28,29]$ and manufactured polymer films [30] with conductive coatings. PDMS is viscoelastic surmising that at long stream times or high temperatures it acts like a gooey fluid like nectar. Regardless of at short stream times or low temperatures, it goes about as a versatile strong like flexible. As needs are, if some PDMS is left on a surface medium-term long stream time it will stream to cover the surface and structure to any surface distortions. Be that as it can if the proportionate PDMS is fell into a circle and flung onto a similar surface short stream time it will influence like an adaptable ball [31].

PDMS is a promising polymer as a covering in light of its high adaptability low surface criticalness negligible exertion inconceivable biocompatibility creation dormancy and straightforwardness [32]. It besides gives two or three positive conditions including high announcement rate consistency controlled thickness colossal scale and folio free contact [33]. On the other hand, de et al. 2009 clarified that the direct conductive film with AgNWs has puzzling adaptability and that go-to move fabricating philosophy can guarantee immaterial effort creation [34, 35]. Although these sensors are electrically conductive and have high affectability the accommodating strain degree of such sensors is still little which confines their presentations. It remains a test to make strain sensors with an enormous accommodating strain range and high affectability [36, 37]. Additionally it is hard to utilize a yarn alone as a wearable sensor and a blend of the yarn with other versatile materials is a significant part of the time required. Nylon as a kind of created fiber has phenomenal prolongation and uncommon security from weariness and substance breaking down and is regularly utilized in the material business [38-40]. Adaptable and wearable electronic devices have destroyed in noteworthy thought because of their uncommon potential practices in areas, for instance, wearable introductions; sharp bits of clothing human improvement and flourishing screen [41-43] in the increase high-imperativeness molecule meds require workmanship contraption and movement [44].

PDMS is interfacing with the response for the production of microfluidic contraptions for normal applications because of it's on a very basic level drawing in properties [45-48]. PDMS if all else fails is an adaptable polymer easy to control biocompatible water impermeable gas powerless optically quick and poor. What's more, PDMS based microfluidic little scale devices grant cementing in a monetarily sharp manner a whole deliberate methodology diminishing its anomaly time of examination test and reagents volumes. A few vocations of PDMS-based cut back scale contraptions are accessible in the blend from the indicated lab-on-a-chip for instance devices for cells culture and examination [49] nuclear tests for the examination of nucleic acids $[50,51]$ and proteins $[52]$ to the alleged organ-on-a-chip [53-56]. The last referenced later application relies on microfluidic structures for the lifestyle of various cells imitating organs and complex tissues [57-60]. The promising features of PDMS cut back scale devices, in any case, are not completely reviewed especially in the perspective of understanding a biomedical insignificant rapid easy-to-use microfluidic sub-nuclear test. A few utilized in the biomedical field could believe it or not advantage by a sub-nuclear test subject to microfluidic contraptions. In this particular circumstance, a PDMS little scale gadget was beginning late made by our gathering for the cleansing and direct presentation of nucleic acids [61-64]. This contraption is made out of a downsized scale chamber which is made by the party of a structure cast globule shaped gap and a PDMS support layer gotten by turn covering.

In this examination endorsing of our PDMS little scale the gadget is showed up at first on obtained examinations, for instance, genotyping and pathogen seeing check to show that, for the most part, used nuclear structures, for instance, steady and multiplex PCR can benefit by the use of a PDMS more diminutive scale contraption. Additionally, 
since PDMS reasonably encounters to surface functionalization $[65,66]$ therefore in this examination basic and naturally kind jump "Dip and Dry" frameworks for the pretreatment of the material surface. Here we advance a reasonable and pragmatic strategy process technique to deliver AgNWs adaptable direct cathode. A uniform course of action of interconnected AgNWs was made on a silicon water by utilizing a drop-covering system. The sub-atomic weight and fixation acknowledge an essential occupation. The morphology and estimation of AgNWs and the favored introduction headway fragment of AgNWs is additionally talked about quickly [67]. Taking a gander at clear films show high transmittance and low sheet square. It will be addressed in this examination that by mishandling PDMS as a host cross segment a colossal number of the weights uncovered AgNWs like the poor bond surface ruthlessness and poor automated belongings will be settled and amazingly conductivity will be improved in the interim. Furthermore, the impact of morphology parameter on straightforwardness and conductivity of the AgNWs arrange have been investigated and new outcomes have been acquired. The electromechanical execution and strain perceiving properties of the FSSF were comparably investigated. This outcome demonstrates that the AgNWs/ PDMS verified surface has a prominent application potential as strain sensors for wearable electronic contraptions.

\section{Materials and methods}

\subsection{Materials}

In this examination, White knitted nylon/PU surfaces $\left(138 \mathrm{~g} / \mathrm{m}^{2}\right), 97 \%$ nylon and $3 \%$ PU were used. The nylon/PU focus spun composite yarn; the liner thickness of composite yarn is $80 \mathrm{D}$. The rib thickness and course thickness of weaved nylon/PU surfaces are $280 / 5 \mathrm{~cm}$ and $140 / 5 \mathrm{~cm}$. The nylon/PU surface tests are cut into $2 \mathrm{~cm} \times 4 \mathrm{~cm}$. The high gauge of AgNWs with a thickness of $40 \mathrm{~nm}$ and dimensional length of 20-60 $\mu \mathrm{m}$ procured from Nanjing XFNANO INC Advanced Materials Supplier Ltd., China and SYLGARDRR 184 SILICONE ELASTOMER $(1 \mathrm{~kg})$ and SYLGARD 184 SILICONE ELASTOMER CURING AGENT $(0.1 \mathrm{~kg})$ acquired from Dow Corning is an enrolled trademark of Dow Corning Corporation.

\subsubsection{Modification of nylon/PU fabric texture with AgNWs}

AgNWs were joined subject to the essential "Dip and Dry" Process. In the essential case, the nylon/PU surfaces at first cleaned in $\left(\mathrm{CH}_{3}\right)_{2} \mathrm{CO}\left(\mathrm{C}_{3} \mathrm{H}_{6} \mathrm{O}\right)$, ethanol $\left(\mathrm{C}_{2} \mathrm{H}_{6} \mathrm{O}\right)$ answer for $30 \mathrm{~min}$ and washed by deionized water for $15 \mathrm{~min}$ and after that dried for $6 \mathrm{~h}$ in the vacuum. Additionally, Nylon/ PU Fabric tests were dunked into $30 \mathrm{~mL}$ AgNWs suspensions with different centers $(1,2,3,4$ and $5 \mathrm{mg} / \mathrm{mL})$ and kept the sample in ultrasonic clearer for $1-5 \mathrm{~h}$ at $25^{\circ} \mathrm{C}$ temperature. Starting there forward, the surfaces were dried at room temperature for $8-24 \mathrm{~h}$, avoid the light. The surfaces changed to silver shading from white due to adsorption of AgNWs course of action. Finally, five cycles of "Dip and Dry" were performed to procure the AgNWs treated surfaces.

\subsubsection{PDMS electrode fabrication of AgNWs}

For PDMS readiness we utilized SYLGARDRR 184 SILICONE ELASTOMER $(1 \mathrm{~kg}) 30 \mathrm{~g}$ and SYLGARD 184 SILICONE ELASTOMER CURING AGENT $(0.1 \mathrm{~kg}) 1.5 \mathrm{~g}$. At that point blending them tenderly and restored, firstly, if, in the Ultrasonic Cleaner, and, secondly, in the, vacuum at $25^{\circ} \mathrm{C}$ for $30 \mathrm{~min}$. After that made a layer of PDMS Film on the two sides of the, PDMS/Nylon/Pu/PDMS, sandwich was relieved at $400^{\circ} \mathrm{C}$ for $24 \mathrm{~h}$ in the dryer. After the AgNWs/PDMS film $(20 \times 20 \mathrm{~mm})$ on SILICON cut and afterward stripped off from the SILICON substrate (Table 1).

The depiction of extraordinary, nylon/PU fabric surface and AgNWs secured nylon surface have shown up in Fig. 1. It might be seen from Fig. 1a the primary nylon surface is diminished. Though, the shade of the nylon/PU fabric superficial improved with AgNWs is silver shading as a display in Fig. 1b in light of the fact that AgNWs shapes a film on the nylon/PU fabric exterior. The shade of the PDMS with AgNWs secured nylon/PU fabric external is Ash due to the declaration of the PDMS and AgNWs Nanoparticles Fig. 1c.

AgNWs were stacked on the surface by methods for "dip and dry" procedure. The method was reiterated on
Table 1 PDMS sample preparation

\begin{tabular}{llllll}
\hline $\begin{array}{l}\text { No } \\
\begin{array}{l}\text { PDMS and curing } \\
\text { agent mass fraction, } \\
\text { mf }\end{array}\end{array}$ & $\begin{array}{l}\text { Curing tem- } \\
\text { perature, Tc }\left({ }^{\circ} \mathrm{C}\right)\end{array}$ & $\begin{array}{l}\text { Curing time, } \\
\text { tc (min) }\end{array}$ & $\begin{array}{l}\text { Vacuum, } \\
\text { time, tv (min) }\end{array}$ & $\begin{array}{l}\text { PDMS/Nylon/Pu/ } \\
\text { PDMS, sandwich dry- } \\
\text { ing time }\end{array}$ \\
\hline 1 & $10: 0.5$ & 25 & 30 & 30 & $40^{\circ} \mathrm{C} \& 24 \mathrm{~h}$ \\
2 & $15: 1.5$ & 25 & 30 & 30 & $40^{\circ} \mathrm{C} \& 24 \mathrm{~h}$ \\
3 & $20: 2$ & 25 & 30 & 30 & $40^{\circ} \mathrm{C} \& 24 \mathrm{~h}$ \\
4 & $25: 2.5$ & 25 & 30 & 30 & $40^{\circ} \mathrm{C} \& 24 \mathrm{~h}$ \\
\hline
\end{tabular}



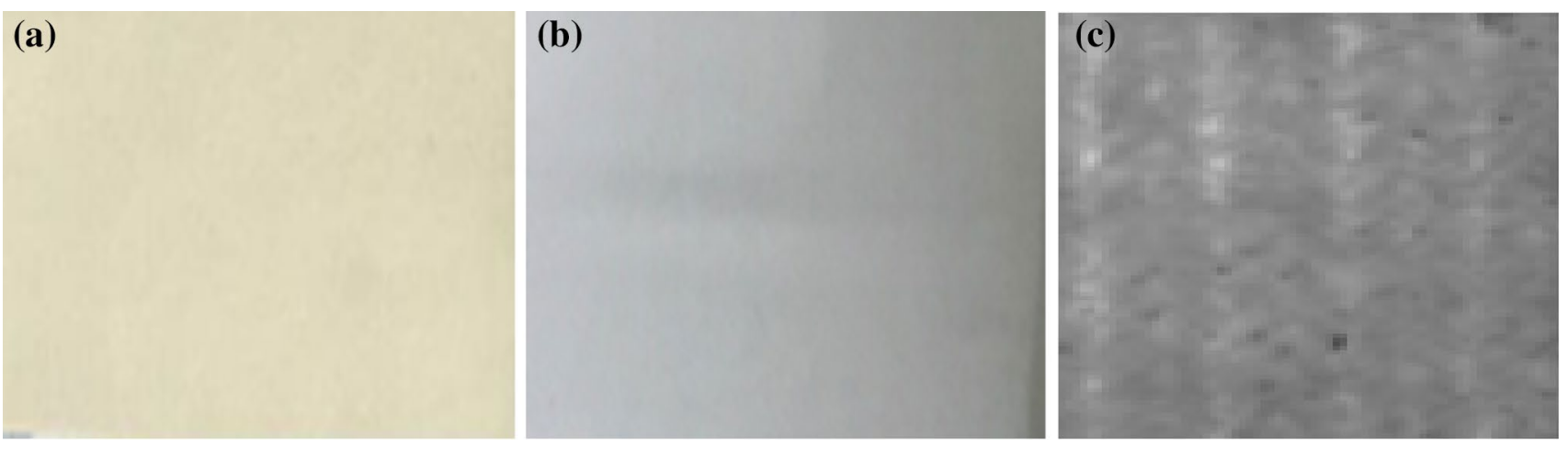

Fig. 1 (a) Pristine nylon/PU fabric, (b) Ag nanowire treated fabric, (c) PDMS sandwich with AgNWs covered nylon/PU fabric

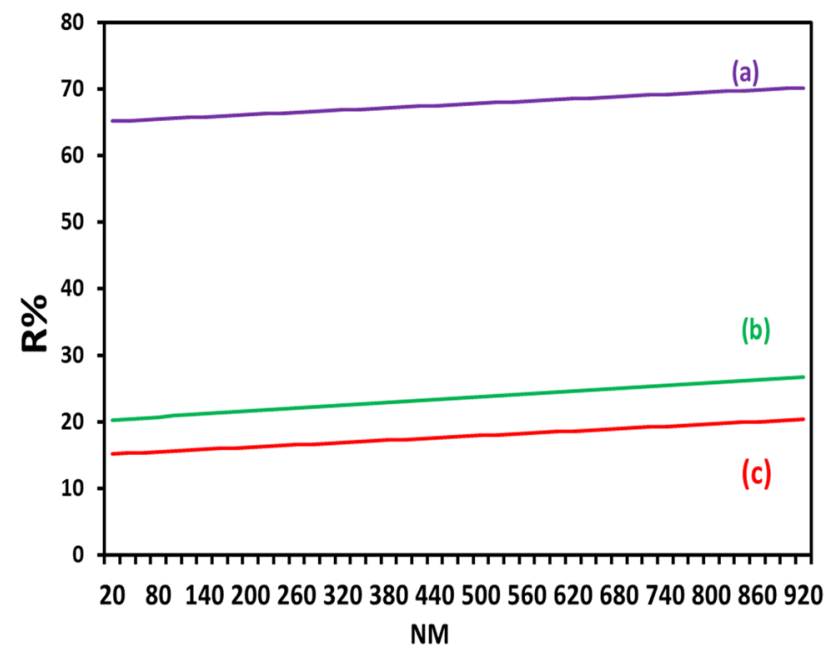

Fig. 2 Reflection spectra of (a) Pristine nylon/PU fabric perfect $65 \%$ and (b) AgNWs-stacked fabric 20\% (c) PDMS film 15\% sandwiched with AgNWs-stacked fabric

different occasions to manufacture a proportion of AgNWs stacking. After the covering, the principal nylon/PU fabric surface changed its shading from white to green due to the silver shade of the AgNWs colloidal course of action. The reflectance spectra of faultless and AgNWs-stacked surfaces in the extent of $630-770 \mathrm{~nm}$ have shown up in Fig. 2. The reflectance dimension of perfect surface decreased from about 65 to $20 \%$ and $15 \%$ in light of the AgNWs stacking and PDMS Film.

\subsubsection{Methodology}

In our examination, we utilized the "Dip and Dry" technique for the texture adjustment and straightforward film covering strategy for optical perspective on test texture. The purpose of present work is to fabricate pristine nylon/ PU fabric with AgNWs, according to its multiple properties, by a one-step simple "dip and dry" treatment. Here, required low-surface energy which makes the "dip and dry" technique easily accessible. There are distinctive strategies are accessible yet we picked "Dip and Dry" as a result of this technique is low expensive and enviro-accommodating.

\subsubsection{Characterization}

Shading estimation of the first example and the silver covered texture test was completed by Raman spectra utilizing a CCD camera with a coordination time of $10 \mathrm{~s}$ by one sweeps. The substance arrangement of the silver covered texture scanning electron microscopy (SEM) estimations was executed by the TESCAN MIRA3 field outflow SME. The electrical resistivity of detached texture example was overviewed by an advanced multi-meter (Key Sight Truevolt 34465A). The Fabric example were clear by two insulators and the measure length was flexible. The mechanical element was observed utilizing an Instron Model 5566 Material Testing framework and Tensile strength of single texture example was estimated. Forward-looking infrared (FLIR) cameras for characteristic percentages difference between ambient and scene high temperature. HARKESPCAX1 for investigate water contact angle.

\section{Results and discussion}

\subsection{SEM characterization}

In order to make a super capacitor \& conductive layers, both distribution \& dispersion of particles are the key factors. The SEM images revealed the relation between electrical conductivity of the fabric \& AgNWs concentration. SEM images of the consolidated AgNWs at different intensification have shown up in Fig. 3, where Fig. 3a pristine nylon/PU fabric, Fig. 3b-d Ag nanowire treated fabric at different magnifications. It will, in general, be seen that the AgNWs are homogenous in morphology. Here, pristine

\section{SN Applied Sciences}



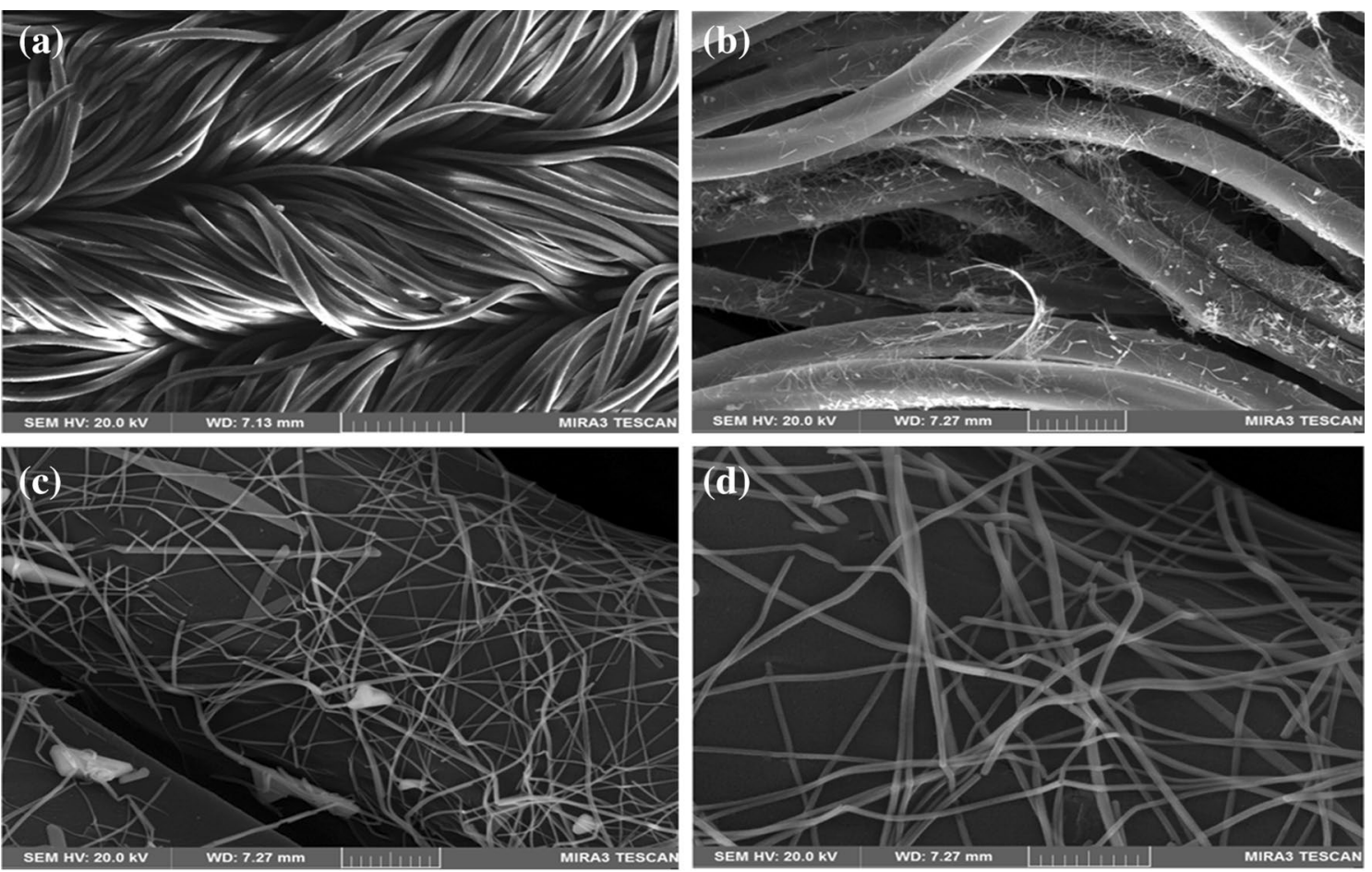

Fig. 3 SEM images of the (a) Pristine nylon/PU fabric, (b-d) Ag nanowire treated fabric at different magnifications

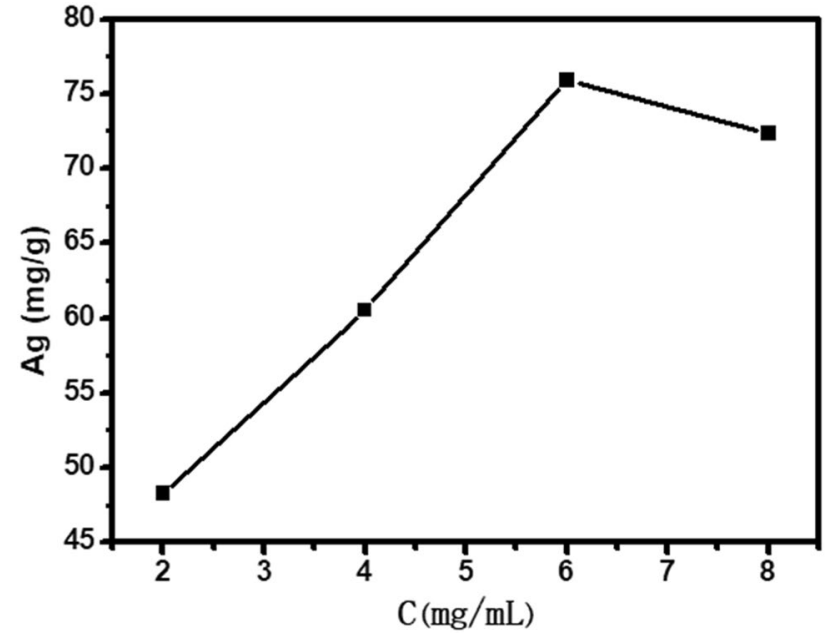

Fig. 4 Concentration (mg/mL) Vs AgNWs-stacked Ag (\%) percentage on the surface of the nylon/PU fabric

nylon/PU fabric were occupied by AgNWs which caused the formation of a nanolayer on the fabric surface.

\subsection{Concentration for AgNWs}

The Fig. 4 shows the concentration vs percentage of AgNWs stacked on the surface of nylon/PU fabric. With the increase of concentration, the percentage is also be increased and after that decreased. Here, four different concentration of AgNWs were used. The best result comes from $6 \mathrm{mg} / \mathrm{mL}$. EDX spectroscopy analyzed the substance association of the outside of the fabric surfaces \& calculated the percentages of AgNWs-stacked Ag (\%). It demonstrated that how many percentages of AgNWs stacked on the surface of nylon/PU fabric. Low-enhancement pictures show that AgNWs-stacked surface had all the earmarks of resembling unsullied one. The high-enhancement pictures reveal a smooth longitudinal fabric structure of immaculate nylon/PU strands. While treated nylon/PU fabric was secured with AgNWs. Plainly, AgNWs secured on the nylon fabric surface have an incredible relationship with each other.

In Fig. 4, The concentration vs percentage of AgNWs stacked on the surface of nylon/PU fabric were $2 \mathrm{mg} / \mathrm{mL}$, $4 \mathrm{mg} / \mathrm{mL}, 6 \mathrm{mg} / \mathrm{mL} \& 8 \mathrm{mg} / \mathrm{mL}$ Vs $47.5 \%, 62.5 \%, 75.5 \%$ \& $72 \%$ respectively. EDX Semi-quantitative examination of AgNWs-stacked surface showed the weight dimension of $75.5 \%$ for AgNWs. The proximity of the AgNWs apex demonstrates the compelling covering of AgNWs outwardly of the fabric surface. To make an electro conductive material, a layer of conductive covering must be accessible to the fiber surface. Stacking of AgNWs to the fiber surfaces changed over the nylon/PU surface into an electro conductive substrate, obviously as a result of the conductive ways formed by the AgNWs interconnected with each other. To achieve a better than the average dimension of surface electrical resistivity of AgNWs electro conductivity, 
the covering method was reiterated on different occasions. The regularly secured nylon surface was assessed to be $3.53 \mathrm{~K} \Omega / \mathrm{cm}$ and showed no enormous differentiation among tests on either side.

The functionalized surface is totally versatile and AgNWs layer can't frail and parts viably under curving weight. The conductivity regard does not change after kept curving. The unique framework structure and high electrical and wear check properties of AgNWs-stacked surface have exceptional potential for use in versatile and stretchable equipment and distinctive devices.

\subsection{The conductive layer of AgNWs on the fabric surfaces}

The AgNWs coated nylon/PU conductive fabric shows different surface electrical resistance for different concentration of AgNWs (Fig. 5a) and soaking time (Fig. 5b).

The proportion of spared AgNWs on the nylon/PU surfaces would direct impact the electrical conductivity of the surface. Figure $5 \mathrm{~b}$ shows the surface restriction essentially lessened when the AgNWs obsession extended to 1 from $2 \mathrm{mg} / \mathrm{mL}$. The flawless nylon/PU surfaces demonstrated unfathomably high surface electrical resistance $(\sim[10] \wedge 6 \mathrm{~K} \Omega / \mathrm{cm})$. At the moment that the AgNWs obsession is $3 \mathrm{mg} / \mathrm{mL}$, the typical estimation of surface electrical restriction for AgNWs secured nylon/PU surface was extended to Fig. 6a $1.79 \mathrm{~K} \Omega / \mathrm{cm}$,
Fig. 5 (a) Surface electrical resistance of AgNWs coated nylon/PU conductive fabric for different concentration of AgNWs. (b) Surface electrical resistance of AgNWs coated nylon/PU conductive fabric for different soaking time
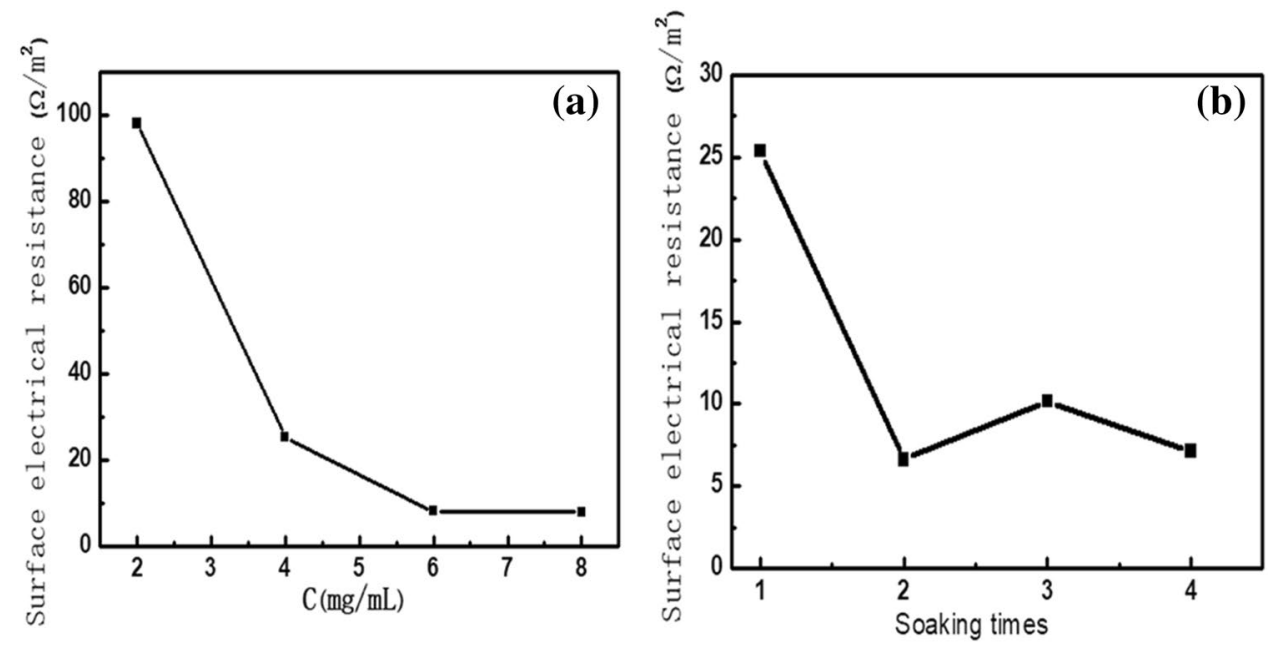

Fig. 6 Surface electrical resistance of $3 \mathrm{mg} / \mathrm{mL}$ for AgNWs coated nylon/PU conductive fabric was (a) $1.79 \mathrm{~K} \Omega / \mathrm{cm}$, (b) $2.97 \mathrm{~K} \Omega / \mathrm{cm}$, (c) $3.53 \mathrm{~K} \Omega / \mathrm{cm}$ and (d) $4.08 \mathrm{~K} \Omega / \mathrm{cm}$
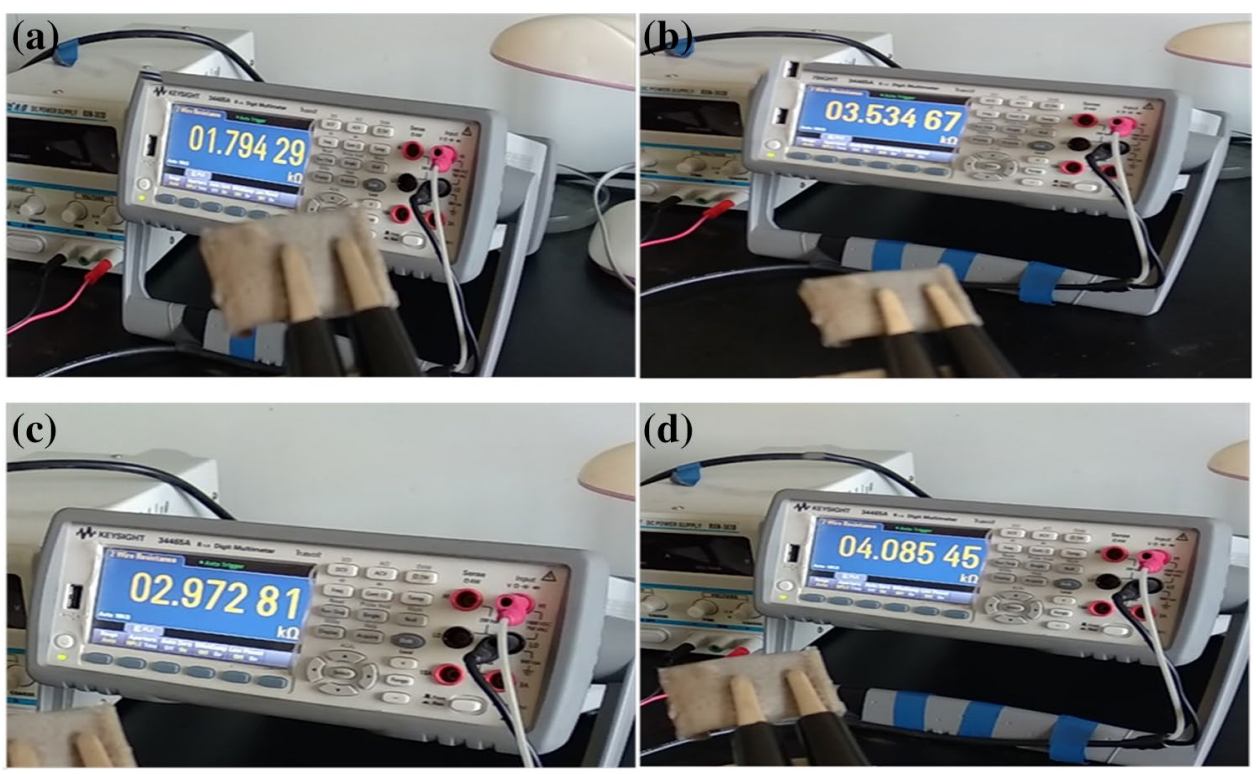
(Fig. 6b) $2.97 \mathrm{~K} \Omega / \mathrm{cm}$, (Fig. 6c) $3.53 \mathrm{~K} \Omega / \mathrm{cm}$ and (Fig. 6d) $4.08 \mathrm{~K} \Omega / \mathrm{cm}$ for $1 \mathrm{~min}, 2 \mathrm{~min}, 3 \mathrm{~min}$ and $4 \mathrm{~min}$ soaking time respectively.

Right when the AgNWs obsession is higher than 4-5 $\mathrm{mg} / \mathrm{mL}$, the surface restriction of AgNWs/nylon/ PU surfaces would, as a rule, be unfaltering, which may be a result of the way that the nylon/PU surface can't assimilate more AgNWs. Along these lines, we picked the AgNWs/nylon/PU surfaces got from $3 \mathrm{mg} / \mathrm{mL}$ of AgNWs obsession to explore the electromechanical property of FSSF.

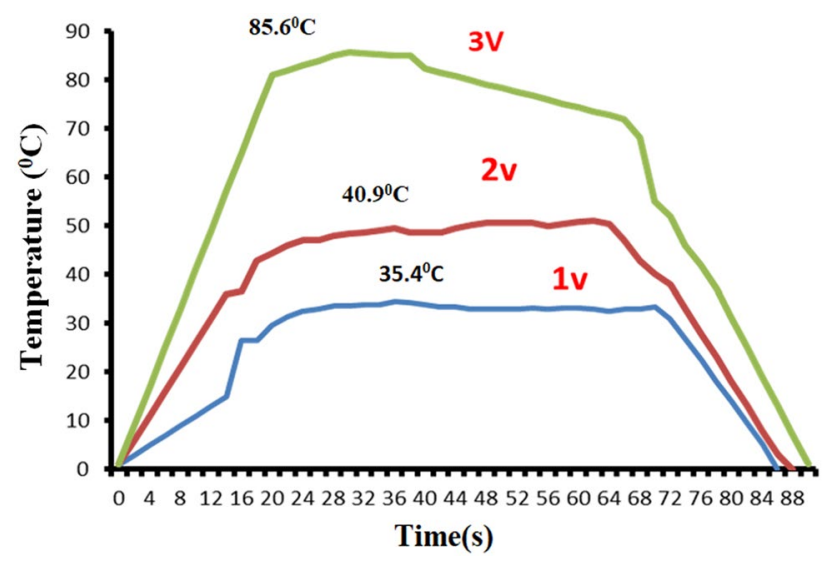

Fig. 7 The temperature measurements during current flow results for the AgNWs coated conductive fabric during constant voltage the FLIR showing highest temperature $35.4^{\circ} \mathrm{C}$ At $1 \mathrm{~V}, 40.9^{\circ} \mathrm{C}$ At $2 \mathrm{~V}$, $85.6^{\circ} \mathrm{C}$ At $3 \mathrm{~V}$ during current flow

\subsection{The temperature and color estimations for conductive fabric}

The surfaces for temperature estimation are two thermistors and a power source with a consistent voltage of $1 \mathrm{~V}$, $2 \mathrm{~V}, 3 \mathrm{~V}$ associated (Figs. 7 and 8). The AgNWs secured conductive surface with minimal released control warm-up more step by step and at the unquestionable moment, the climbing of temperature comes to a standstill while the AgNWs secured conductive surface with set up higher power warm-up quickly and could keep warming up when the voltage is extended. The resistive part scatters all the power by warmth.

Along these lines, the warm-up temperature and warmup time can be adjusted by the assurance of fitting voltage to AgNWs secured conductive surface. Thus, the visual appearance and effortlessness of particular execution, similarly as smoothness of warming and giving out warmth, transform into the most basic assurance criteria of AgNWs secured conductive surface. The edges and the focal point of the warming segment and the most specific results are seen by the AgNWs secured conductive surface.

For the investigation, single went up against Knitting designs with a figural resistive warming segment Knitted AgNWs secured conductive surface are used; two thermistors and a power source with the perfect picked voltage of $1 \mathrm{~V}, 2 \mathrm{~V}, 3 \mathrm{~V}$ are joined to the precedent with the flow of 14I-22I current (Figs. 7 and 8 ).

Preliminary outcomes with temperature estimations of three repeated estimations are showed up in outlines (Fig. 9). Each warming zone of the two zones is warmed and evaluated autonomously. By analyzing the results, it is seen that every sort of AgNWs secured conductive fabric
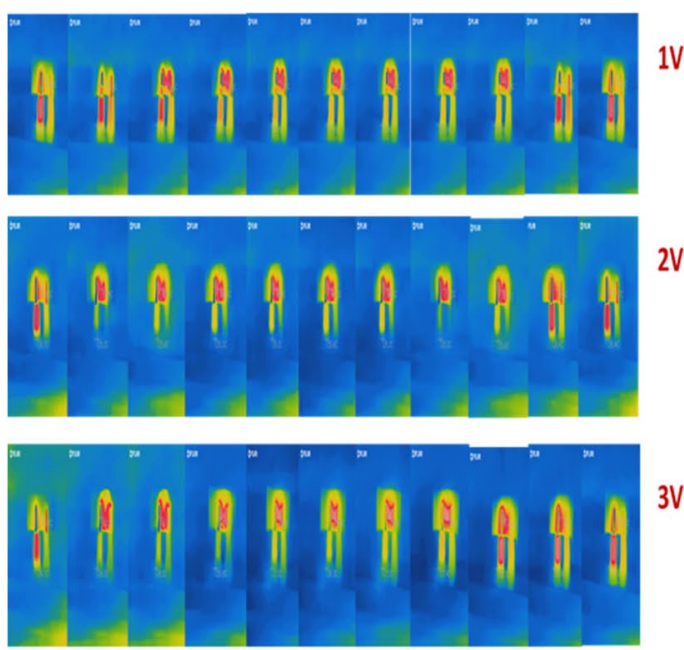

IV

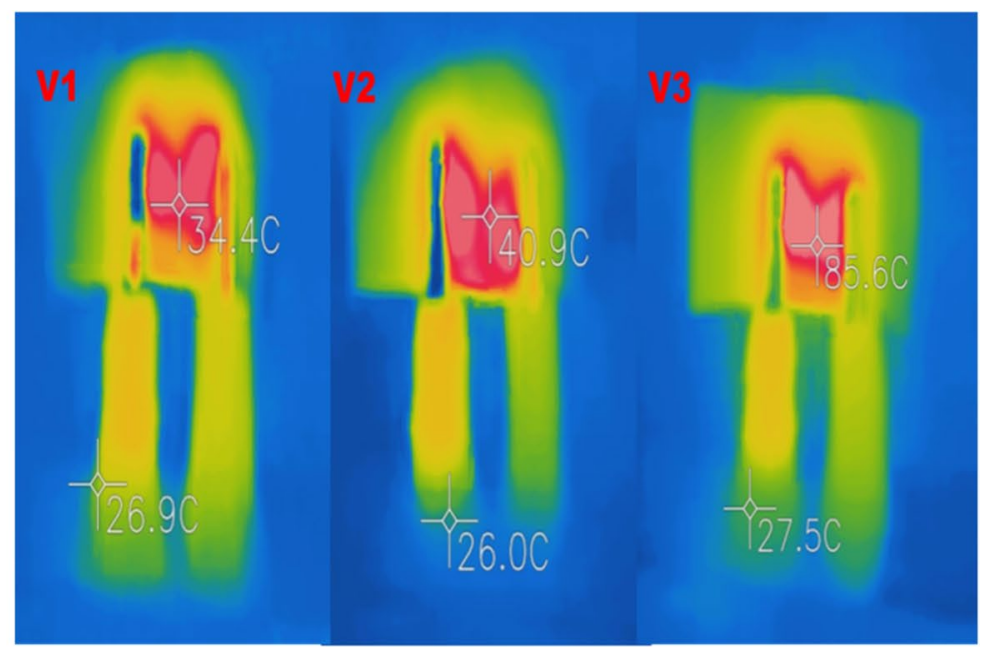

Fig. 8 The knitted AgNWs coated conductive fabric for temperature $\left({ }^{\circ} \mathrm{C}\right)$ measurement during constant voltage of $1 \mathrm{~V}, 2 \mathrm{~V}, 3 \mathrm{~V}$ current flow by FLIR image 


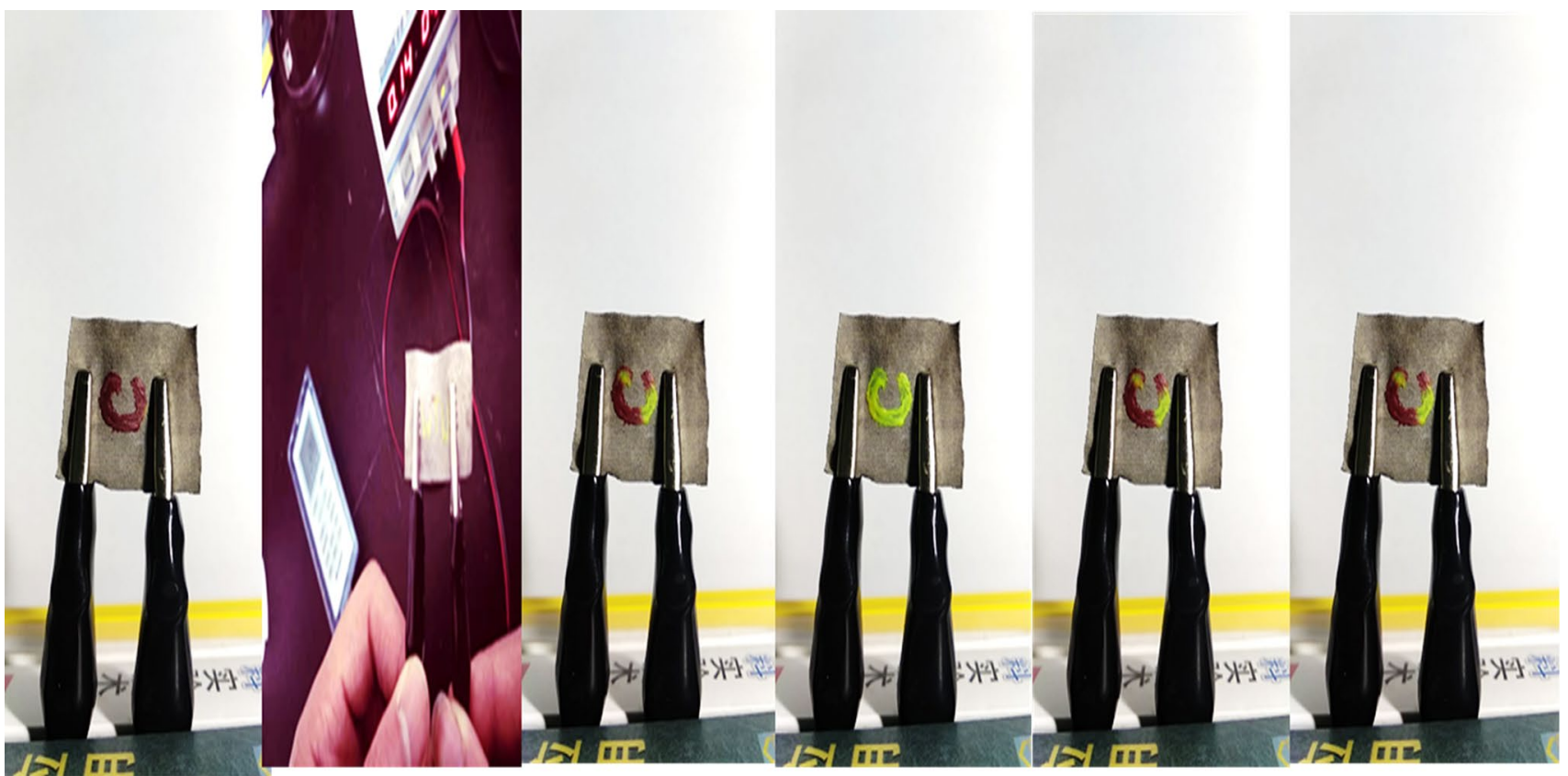

Fig. 9 The equipment for temperature measurements of a figurative knitted AgNWs coated conductive fabric heating for changing colors of temperature variable ink red to yellow

surface has its most outrageous reachable temperature which is released at a predictable voltage. The diagrams show that the temperature rises and by achieving the most extraordinary characteristics it modifies itself. On justification of this investigation, the AgNWs secured conductive surface is considered as the most suitable for the gathering of a resistive warming segment with figural weaved AgNWs secured conductive zone; the segment heats up to at a voltage of $1 \mathrm{~V}, 2 \mathrm{~V}, 3 \mathrm{~V}$ to the temperature of $35.4^{\circ} \mathrm{C}$, $40.9^{\circ} \mathrm{C}, 85.6^{\circ} \mathrm{C}$ in less than 1 min yet they the warming the temperature could accomplish $90^{\circ} \mathrm{C}$. Such temperature is sufficient to make pleasant warmth parts changing colors of temperature variable ink red to yellow. By changing the color, it ensured the stabilization of comfortable heating temperature.

\subsection{Confirmation of restriction changes amidst the strain of the warming portion}

To build up the obstruction changes under the store, the conductive surface is encouraged by AgNWs covering into the surface and the extending test is done (Fig. 10). The object of this test is to discover how the electrical limitation of knitted fabric in the surface test piece changes by a minor piece at any given moment developing the heat. The deterrent $(\Omega)$ is surveyed in advance and amidst the stretching out the store of $100 \mathrm{~g}$ up to $1000 \mathrm{~g}$. Opposition changes under the heap are not unequivocal (Fig. 11). The estimation results toward the starting display a smart

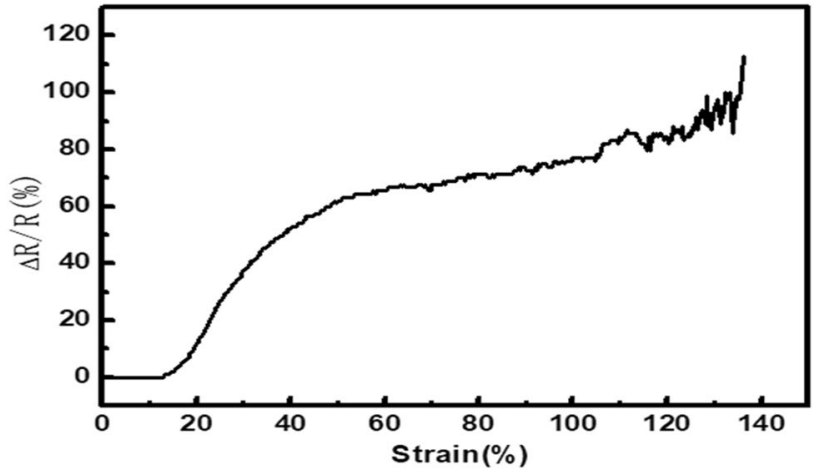

Fig. 10 Resistance variation of AgNWs covered Nylon/Pu fabric during tension

expansion of confinement by a sewed instance of nylon/ Pu fabric surfaces and AgNWs verified conductive zone of treated sewed surface yarns under the store $0-120 \Omega$ and at load and over it is fundamentally elongation length of conductive fabric $35 \%$.

\subsection{Mechanical properties of the versatile strain sensor characteristics}

In this investigation, the plain course nature of precedents with a width of $20 \mathrm{~mm}$ at a check length of $100 \mathrm{~mm}$ was attempted Typical quality strain twists in the midst of stretching out of impeccable fabric surface and AgNWs

\section{SN Applied Sciences}



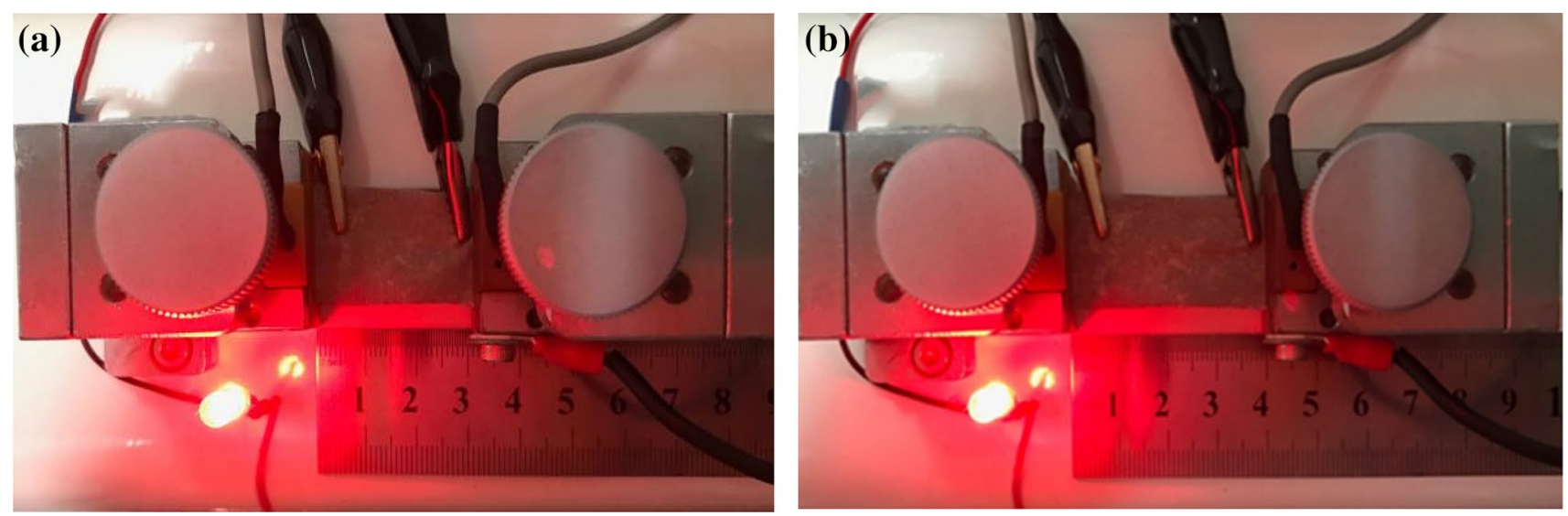

Fig. 11 (a) Original length (b) elongation length of conductive fabric 35\%

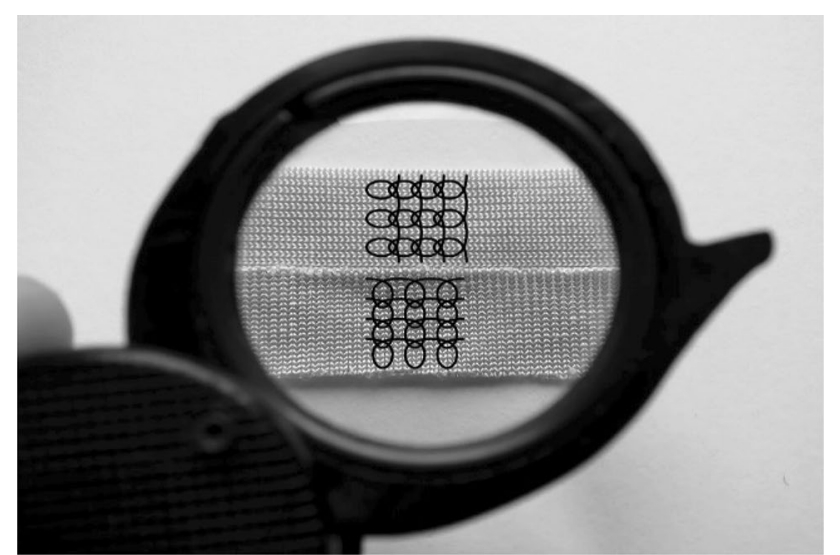

Fig. 12 Illustrations of the knitted structure of nylon/PU fabric

treated consistency (FSSF) are depicted in Fig. 12 strain. The breaking nature of the impeccable surface possibly reduced from 65.3 to $51.7 \mathrm{~N}$ when the nylon/PU fabric surfaces were saved with AgNWs. The AgNWs covering did not add to the improvement of the nature of nylon/PU fabric surface as the AgNWs Nanosheets outwardly of the fabric surface did not outline a thick steady layer. Optical photo of the adaptable strain sensor fabric during (a) $0 \%$ strain, (b) 29\% strain, (c) 35\% strain Fig. 13.

The decrease in quality from the treatment with AgNWs may be a direct result of the corruption of the fabric surface in the treatment technique. In any case, the quality consistency standard of the fabric surface was over $90 \%$. In the meantime, the strain level of consistency of the fabric surface was close $93 \%$. The pliable test results prescribe that the creation system of FSSF did not impact the mechanical properties of the nylon/PU fabric surface which paves the course for practical employments of FSSF.

\subsection{Electromechanical execution of the FSSF}

In the Knitted surface, a "course" of weave is a commonly even line of needle circles and a "grain "of knitting is a fantastically vertical section of interlaced needle circles (Fig. 12). The covering level of AgNWs Nanosheets on nylon/PU fabric surfaces lessened as the surfaces was
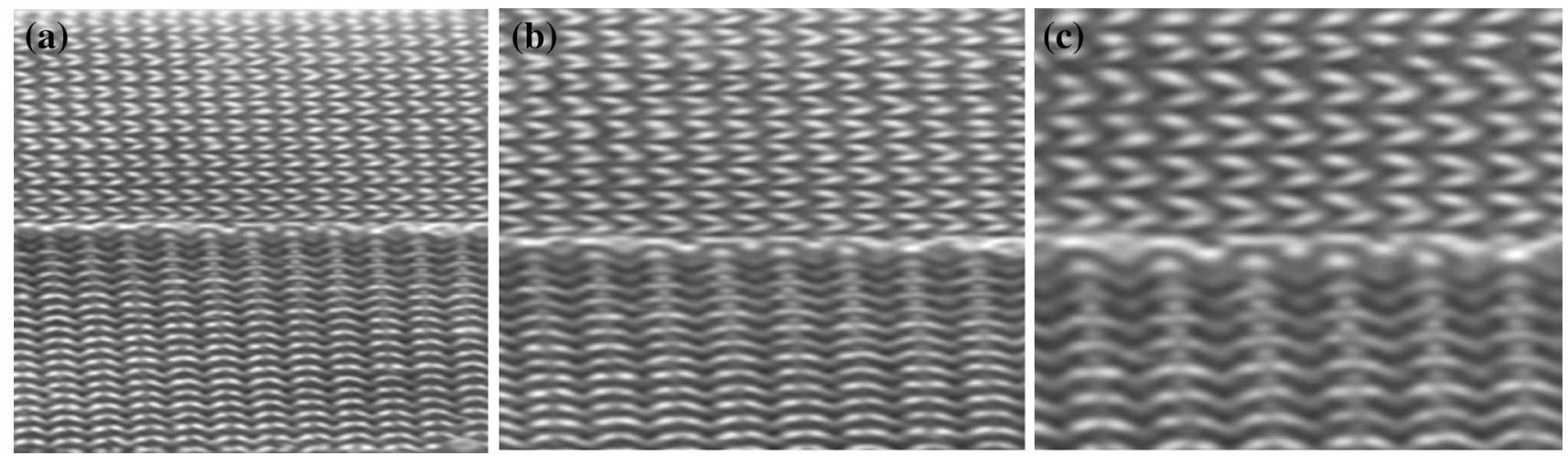

Fig. 13 Optical photograph of the flexible strain sensor fabric during (a) 0\% strain, (b) 29\% strain, (c) 35\% strain 
stretched out to a particular degree (Fig. 13). The photograph shows the optical modest pictures of surfaces identifying with different strain regards and the structures of nylon/PU fabric surfaces were seen doubtlessly. Figure 14a shows the deterrent difference in FSSF for different strains $(5 \%, 12 \%, 20 \%$, and $25 \%)$ under cyclic broadening releasing The resistance change extended with the growth of the strain, which was unsurprising with the results showed up in (Fig. 14). The response of block change to strain under the cyclic expanding and releasing demonstrates the steadfast nature of FSSF. In addition, the effect of flexible speed (strain rate) on the strain identifying properties of FSSF was examined. As can be seen from Fig. 14b, the hindrance change barely extended as the pliable speed extended, which may be because the brief structure response of FSSF is obliged at a high tractable speed. The material surface is anisotropic, inciting a not too bad assortment of strain recognizing properties of the nylon/PU fabric surface in different ways under broadening conditions Fig. 14c demonstrates the strain distinguishing properties of FSSF in wale and course heading at a comparative strain and flexible speed. It will, in general, be seen that the plentifulness and profile of the twists of FSSF in wale and course bearing were noticeably unprecedented. The grain heading demonstrated higher affectability, which could be a result of the higher adaptability of the surface in the edge course. The anisotropic strain distinguishing features of FSSF propelled us to make a fused watching stage and make identifying procedures of tangled developments subject to extraordinary restriction changes in wale and course orientation, While, isotropic fabric surfaces, for instance, nonwoven substrates can't get perceiving recognizing properties in different ways.

In addition, robustness is extraordinarily basic to even minded employments of strain distinguishing nylon/PU fabric. Figure 14d exhibits the check change of FSSF sensor surfaces under 140 cyclic expanding releasing of $5 \%$ suffer frequencies of $0.098 \mathrm{~Hz}$. The electrical response of FSSF showed high dauntlessness to strain

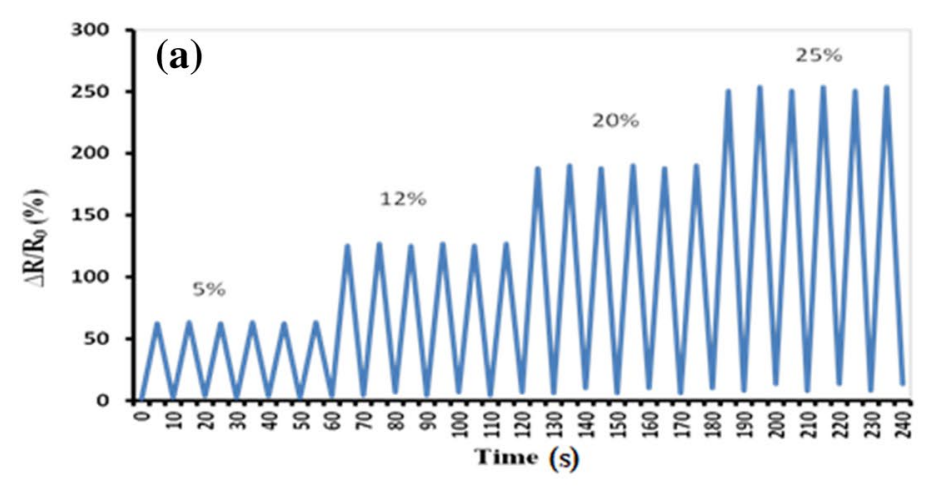

(c)

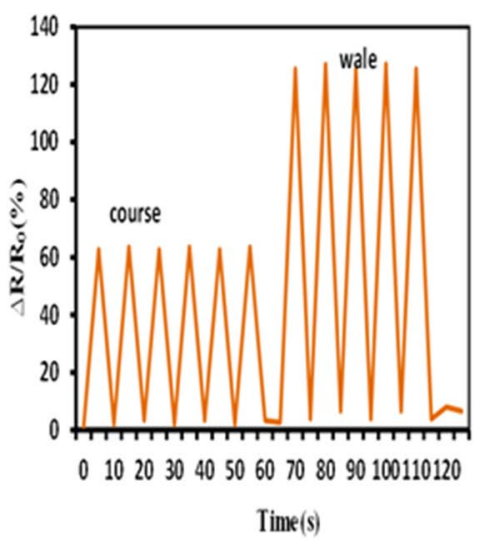

(d)

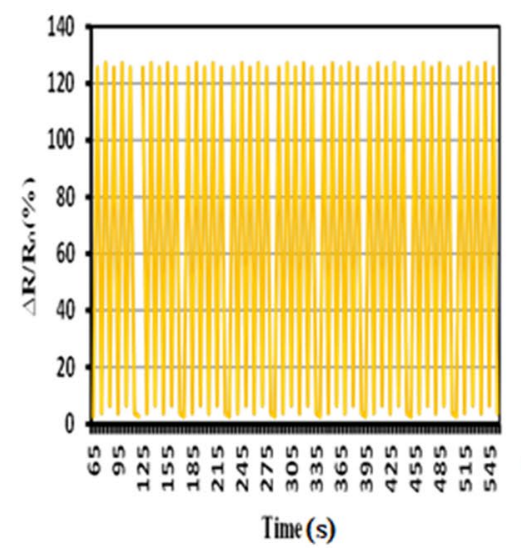

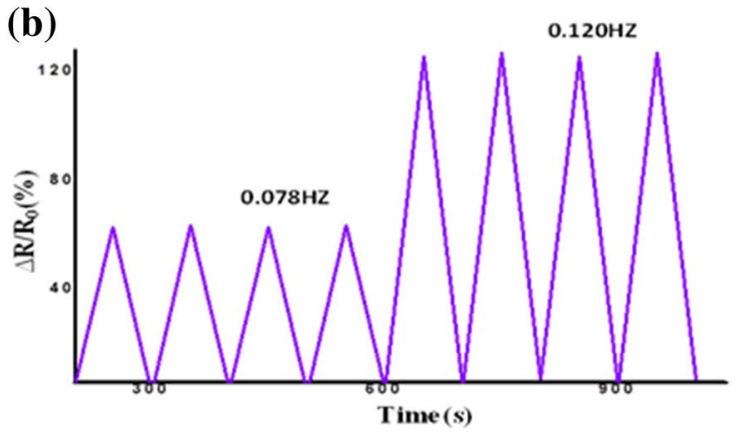

(e)

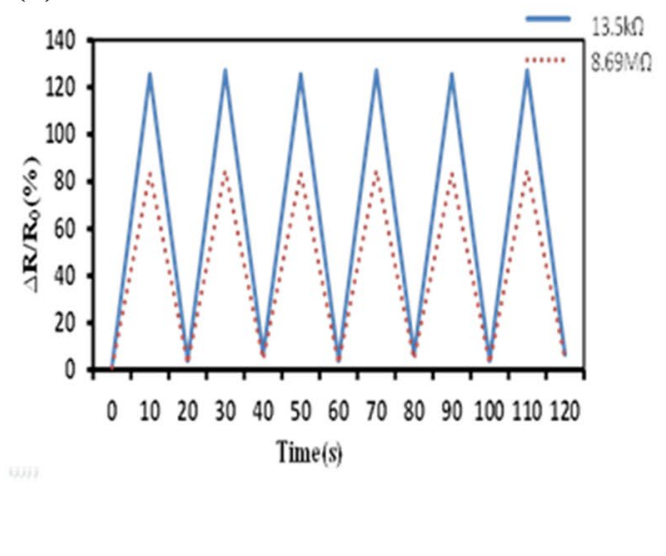

Fig. 14 Electromechanical execution of the adaptable strain identifying surface. (a) relative resistance assortment $\left(\Delta R / R_{0}\right)$ versus cyclic bendable strain of $5 \%, 12 \%, 20 \%$ and $25 \%$; (b) obstacle change under cyclic broadening releasing with a strain of $25 \%$ at frequencies of $0.078 \mathrm{~Hz}$; (c) restriction change of surface in wale and course heading at a repeat of $0.098 \mathrm{~Hz}$; (d) the quality preliminary of FSSF under cyclic flexible strain of $5 \%$ for 140 cycles; (e) relative resistance assortment $(\Delta R / R 0)$ versus cyclic moldable strain of $3 \%$ contrasting with FSSF with different hindrance regards $13.5 \mathrm{~K} \Omega$ and $8.69 \mathrm{M} \Omega$ 
with a low buoy, in the midst of the reiterated cyclic broadening releasing procedure. No detectable hysteresis was found in the plot of relative impediment changes versus strain (inset in Fig. 14d). The results exhibit that the versatile strain recognizing surfaces have whole deal relentlessness and extraordinary strength. In addition, to look into the effect of resistance on identifying features of the FSSF, the block change of FSSF with different restriction regards $(13.5 \mathrm{~K} \Omega$ and $8.69 \mathrm{M} \Omega$ ) under cyclic broadening releasing with a strain of $5 \%$ were watched (Fig. 14e). Differentiating the curves of resistance changes for two FSSFS, the little refinement was found through the block differentiate between the two FSSFS was gigantic. The result surmises that the obstacle of AgNWs/nylon/PU fabric surfaces in a particular range showed a slight effect on recognizing properties of FSSF.

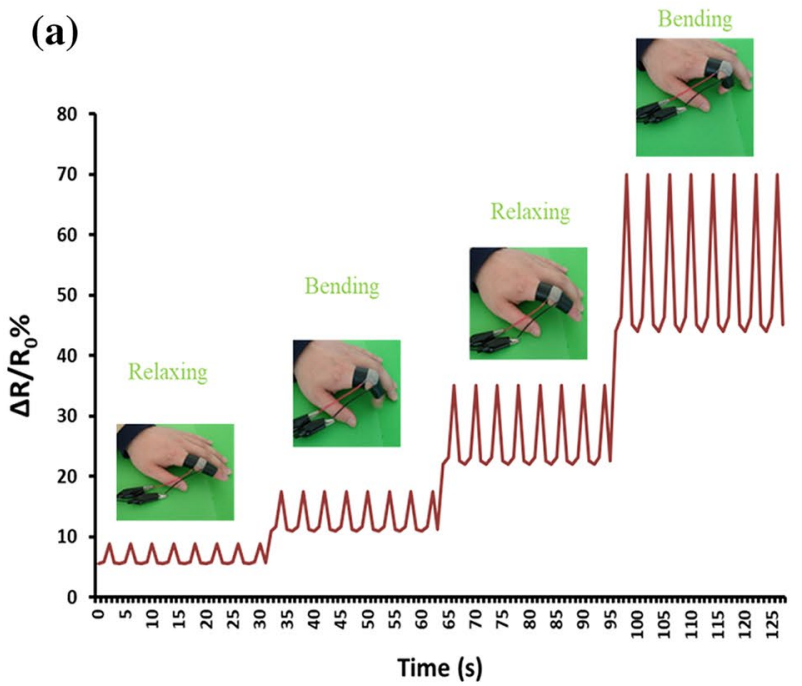

\subsection{Observing of human movements dependent on FSSF}

In order to demonstrate the potential use of FSSF as wearable contraptions, the FSSF was used to screen human development persistently. The FSSF was joined on a finger for distinguishing a minor strain from bowing a finger (inset in Fig. 15a). The bowing developments of the finger were unequivocally checked by account the hindrance change of FSSF. Right when the finger wound to a particular edge, the resistance changes of strain sensor distinctly extended, and a short time later remained stable. In this examination, we used FSSF to perceive both the flexion and unrest of the wrist. The power of twist of the relative resistance change extended as the wrist flexed and a short time later returned to the basic measurement after the wrist restored to its interesting status (Fig. 15b). The plenitude and condition of the resistance twist changed with the edge and speed of wrist flexion. In a general sense, on wrist rotate can be seen by account the electrical

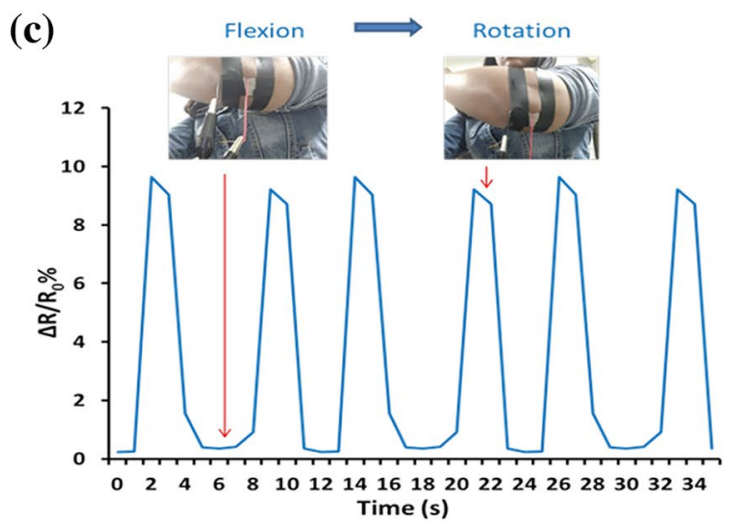

Fig. 15 Monitoring of the Human movement amid (a) Finger Relaxing and twisting, (b) Wrist flexion and revolution, and (c) Elbow flexion and bowing of Sandwiched PDMS film on AgNWs 
hindrance of FSSF (Fig. 14c). The typical for tops in the curve of electrical resistance should join the effects of anisotropic electromechanical properties of FSSF. The flexion and turn of a wrist can be watched viably by account check changes of FSSF. The wealth and repeat of check curves can distinguish the improvement degree and speed of monitions, independently. These results show the limit of FSSF as wearable devices to screen human developments.

\subsection{Water contact viewpoint}

The Hydrophobicity of the PDMS film on both sides of the PDMS/AgNWs/PDMS Conductive sandwiched surfaces was studied by the water contact point estimation. PDMS film sandwiched with PDMS/AgNWs/PDMS Conductive sandwiched surfaces surface is a naturally hydrophilic surface which can be totally wetted by water in view of the rich hydroxyl bunches in its structure. All things considered, the water contact purpose of the essential nylon surfaces surface is 0.0 (Fig. 16a) and PDMS/AgNWs/PDMS Conductive sandwiched texture surface is $38.4^{\circ}$ (Fig. 16b). In any case, the water contact motivation behind PDMS/AgNWs/PDMS Conductive sandwiched verified nylon surfaces adjusted with PDMS is $126.7^{\circ}$ because of the advancement of its surface unpalatability and the lessening of a void region between the PDMS/AgNWs/PDMS Conductive sandwiched verified nylon surfaces strands as appeared in (Fig. 16c). The outcome displays that PDMS film with AgNWs verified nylon surfaces surface adjusted with PDMS shows hydrophobicity. As demonstrated by the Wenzel appear, fluid touches contacts with the outside of the surface and altogether goes into the melancholy on an awful surface. The "self-evident" contact point application of a liquid globule on a brutal surface is identified with the "veritable" contact edge of the spot on a smooth surface by the awfulness factor ( $r$ ) of the surface as depicted in Eq. (1)

$\cos \theta_{\text {app }}=r \cos \theta_{s}$

where the surface unpalatability factor $(r)$ is depicted as the degree of the geometric surface area to the genuine surface space (Eq. 2)

$r=\frac{\text { Geometric Surface Area }}{\text { Actual Surface Area }}$

The Wenzel condition demonstrates that the hydrophilicity and hydrophobicity of a surface rely on the likelihood of the relating outer. The hydrophilicity of a hydrophilic shallow $\left(<90^{\circ}\right.$ and $\left.r>1\right)$ growths through an improvement in the surface obnoxiousness. All of a sudden, for a hydrophobic surface $\left(>90^{\circ}\right.$ and $\left.r>1\right)$, surface hydrophobicity increments with the move of surface disagreeableness. The water contact point can be passed on by Eq. (3)
Fig. 16 Water contact angle of (a) original, (b) PDMS on both side of AgNWs coated nylon fabric, (c, d) after water drop out
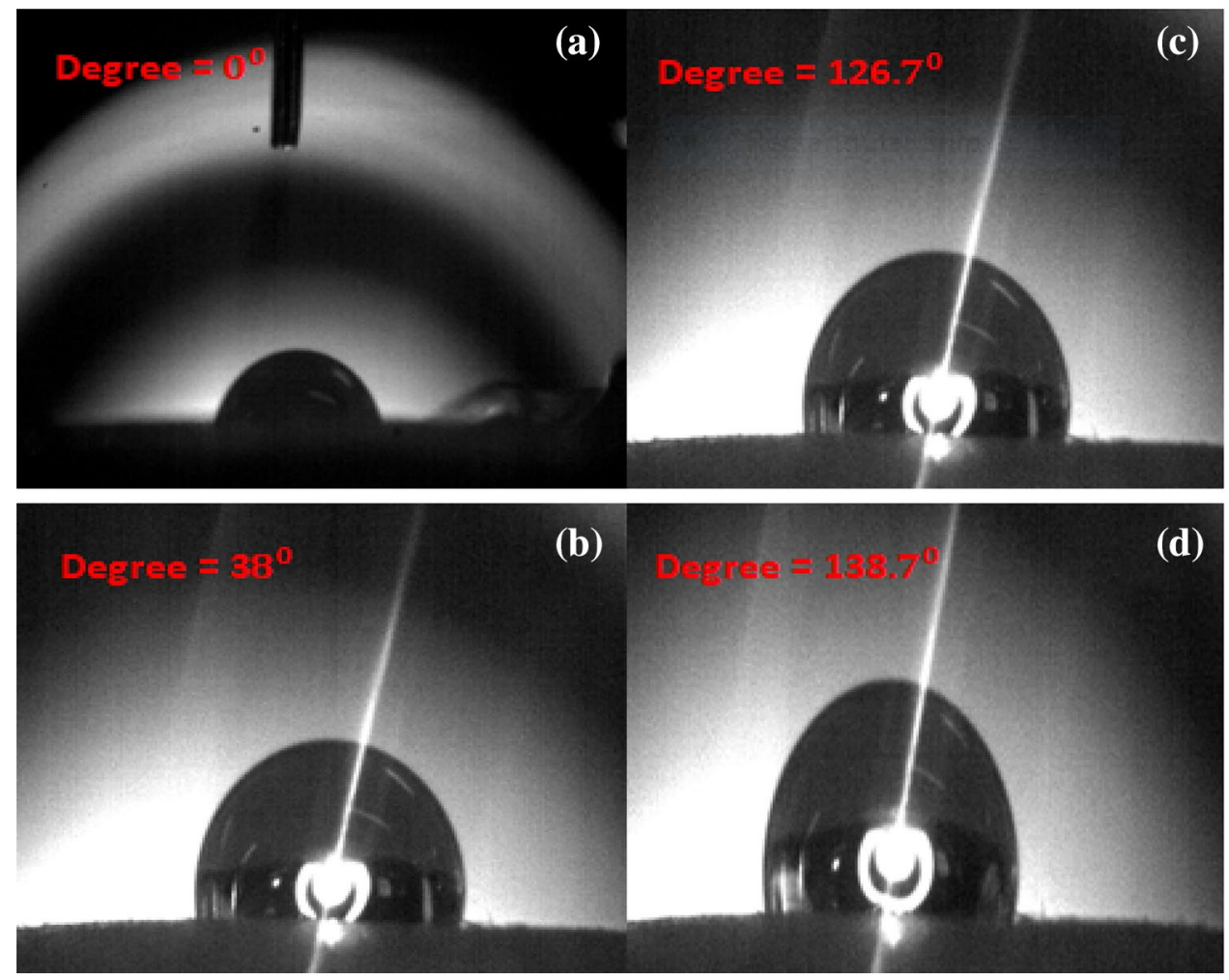
$\cos \theta_{\text {app }}=\frac{\text { Geometric Surface Area }}{\text { Actual Surface Area }} \cos \theta_{s}$

The outside of PDMS/AgNWs/PDMS Conductive sandwiched secured nylon/PU fabric surface adjusted with PDMS is obnoxious as appeared in pictures (Fig. 16c, d). The air that is gotten in the unforgiving surface of irregularities and despondencies on PDMS/AgNWs/PDMS Conductive sandwiched verified nylon/PU fabric surface essentially improves the fabric surface hydrophobicity. What's more, modification of PDMS makes discretionary nanoscale fierceness. In like way, the contact point is extended. The water cooperation edge of the AgNWs verified nylon/PU fabric surfaces with PDMS just decreases from $138.7^{\circ}$ before washing to $126.7^{\circ}$ after washing as appeared in Fig. 16d. The results further authenticate that PDMS film as soon as possible both sides of the AgNWs verified nylon/PU fabric textures adjusted with PDMS has the mind-blowing velocity to water supersede or Hydrophobicity.

\section{Discussion}

Reflection spectra of Pristine nylon/PU fabric perfect $65 \%$ and AgNWs-stacked fabric 20\%, PDMS film 15\% sandwiched with AgNWs-stacked fabric. AgNWs verified on the nylon/PU knitted fabric texture With PDMS film were optional and have an extraordinary association with one another. The immaculate nylon/PU fabric surfaces exhibited incomprehensibly high superficial electrical resistance. Superficial electrical resistance of $3 \mathrm{mg} / \mathrm{mL}$ for AgNWs coated nylon/PU conductive fabric was (a) $1.79 \mathrm{~K} \Omega /$ $\mathrm{cm}$, (b) $2.97 \mathrm{~K} \Omega / \mathrm{cm}$, (c) $3.53 \mathrm{~K} \Omega / \mathrm{cm}$ and (d) $4.08 \mathrm{~K} \Omega / \mathrm{cm}$. The AgNWs verified conductive superficial is considered as the most appropriate for the shared instance of a resistive warming section with figural weaved AgNWs verified conductive zone; the fragment heat up to at a voltage of $1 \mathrm{~V}, 2 \mathrm{~V}, 3 \mathrm{~V}$ to the temperature of $35.4{ }^{\circ} \mathrm{C}, 40.9{ }^{\circ} \mathrm{C}, 85.6^{\circ} \mathrm{C}$ in under $1 \mathrm{~min}$ yet they the warming the temperature could achieve $90^{\circ} \mathrm{C}$ and change materials colors. Such temperature is adequate to make charming warmth parts for a human. For the social affair of a verified warming part, the nylon/PU texture surfaces with AgNWs must be considered as unlawful inferable from gigantic hindrance changes of the resistive zone influenced by the texture of fabric surface weight (to $\sim 100 \Omega$ ). Electromechanical execution of the versatile strain distinguishing surface, relative opposition variety $\left(\Delta R / R_{0}\right)$ versus cyclic bendable strain of $5 \%, 12 \%, 20 \%$ and $25 \%$; deterrent change under cyclic widening discharging with a strain of $25 \%$ at frequencies of $0.078 \mathrm{~Hz}$; confinement change of surface in wale and course heading at a rehash of $0.098 \mathrm{~Hz}$; the quality starter of FSSF under cyclic adaptable strain of $5 \%$ for 140 cycles; relative obstruction combination $\left(\Delta R / R_{0} \%\right)$ versus cyclic flexible strain of $3 \%$ standing out from FSSF with various impediment respects $(13.5 \mathrm{~K} \Omega$ and $8.69 \mathrm{M} \Omega$ ). By analyzing the water contact view point, the AgNWs verified nylon/PU fabric textures adjusted with PDMS has the mind-blowing velocity to water supersede or Hydrophobicity.

\section{Conclusion}

This study described a simple, versatile, and effective approach for creation new functionalities on pristine nylon/PU fabric. The conductive fabric was prepared by the facile "dip \& dry" coating of AgNWs/PDMS films. It was demonstrated that loading of AgNWs on surface of Pristine nylon/PU fabric can convert insulator fabrics into textile based substrate supercapacitor \& conductors. By analyzing different characteristics, It shows as a flexible strain sensor. Expanding turning edge accomplished a further extension in the restriction of FSSF, framing a phase banner, which shows the convenient response and high affectability of FSSF and By morals of the mixed up deforming of human improvement, it is vital to screen the multi-directional advancement attributes by a solitary strain surface sensor and temperature with color changing. PDMS film as quickly as time permits the two sides of the AgNWs tested nylon/PU fabric surfaces balanced with PDMS has the awesome speed to water replace or Hydrophobicity. The FSSF as a productive flexible strain sensor will bolster the improvement of novel wearable electronic contraptions. This effort gives a novel strategy to figure out how to gather conductive extraordinary and adaptable attributes instead of potential application in a thing of genuine apparel.

\subsection{Future plan}

This examination portrayed a fundamental adaptable and stunning procedure for the creation of new functionalities on nylon/PU texture surfaces fixings. The FSSF as a productive flexible strain sensor will bolster the improvement of novel wearable electronic contraptions. This effort gives a novel strategy to figure out how to gather conductive extraordinary and adaptable attributes instead of potential application in a thing of genuine apparel. After the slanted arrangement of a layer on the PDMS/AgNWs fabric/PDMS sandwiched surface the absurdly hydrophobic superficial rehabilitated over into the hydrophobic substrate. The improved fabric superficial mercilessness presented through AgNWs-PDMS film and the reduction of the without fabric texture importance actuated through coating responsible for the water $\left(\mathrm{H}_{2} \mathrm{O}\right)$ counteract feature of the 
preserved superficial. In further we can use it in medical textile, Flexible electroconductive device, thermo conductive materials, hydrophobic \& strain sensing materials.

\section{Compliance with ethical standards}

Conflict of interest There is no conflict of interest in this paper.

\section{References}

1. Segura $G$, Guzmán $P$, Zuñiga $P$, Chaves $S$, Barrantes $Y$, Navarro $G$, Asenjo J, Guadamuz S, Vargas VI, Chaves J (2015) Copper deposition on fabrics by rf plasma sputtering for medical applications. J Phys Conf Ser 591(1):012046

2. Yu J, Wu J, Wang H, Zhou A, Huang C, Bai H, Li L (2016) Metallic fabrics as the current collector for high-performance graphenebased flexible solid-state supercapacitor. ACS Appl Mater Interfaces 8(7):4724-4729

3. Amjadi M, Kyung KU, Park I, Sitti M (2016) Stretchable, skinmountable, and wearable strain sensors and their potential applications: a review. Adv Funct Mater 26(11):1678-1698

4. Zeng W, Shu L, Li Q, Chen S, Wang F, Tao XM (2014) Fiber-based wearable electronics: a review of materials, fabrication, devices, and applications. Adv Mater 26(31):5310-5336

5. Weng W, Chen P, He S, Sun X, Peng H (2016) Smart electronic textiles. Angew Chem Int Ed 55(21):6140-6169

6. Wu X, Han Y, Zhang X, Lu C (2016) Highly sensitive, stretchable, and wash-durable strain sensor based on ultrathin conductive layer@polyurethane yarn for tiny motion monitoring. ACS Appl Mater Interfaces 8(15):9936-9945

7. Shao F, Bian SW, Zhu Q, Guo MX, Liu S, Peng YH (2016) Fabrication of polyaniline/graphene/polyester textile electrode materials for flexible supercapacitors with high capacitance and cycling stability. Chem Asian J 11(13):1906-1912

8. Molina J, Zille A, Fernández J, Souto AP, Bonastre J, Cases F (2015) Conducting fabrics of polyester coated with polypyrrole and doped with graphene oxide. Synth Met 204:110-121

9. Lee JY, Connor ST, Cui Y, Peumans P (2008) Solution-processed metal nanowire mesh transparent electrodes. Nano Lett 8(2):689-692

10. De S, Higgins TM, Lyons PE, Doherty EM, Nirmalraj PN, Blau WJ, Boland JJ, Coleman JN (2009) Silver nanowire networks as flexible. ACS Nano 3(7):1767-1774

11. Gonsalves KE, Xiao TD, Strutt PR (1993) Processing and property evaluation of $\mathrm{Si}(\mathrm{N}, \mathrm{C})$ coatings on silica glass/quartz substrates. MRSOnline Proceedings Library Archive, vol 327.

12. Hoipkemeier-Wilson $L$, Schumacher JF, Carman ML, Gibson AL, Feinberg AW, Callow ME, Finlay JA, Callow JA, Brennan AB (2004) Antifouling potential of lubricious, micro-engineered, PDMS elastomers against zoospores of the green fouling alga ulva (enteromorpha). Biofouling 20(1):53-63

13. Chen W, Rakhi RB, Hu L, Xie X, Cui Y, Alshareef HN (2011) Highperformance nanostructured supercapacitors on a sponge. Nano Lett 11(12):5165-5172

14. Ye S, Feng J, Wu P (2013) Deposition of three-dimensional graphene aerogel on nickel foam as a binder-free supercapacitor electrode. ACS Appl Mater Interfaces 5(15):7122-7129

15. Pham VH, Dickerson JH (2016) Reduced graphene oxide hydrogels deposited in nickel foam for supercapacitor applications: toward high volumetric capacitance. J Phys Chem C 120(10):5353-5360
16. Yang C, Gu H, Lin W, Yuen MM, Wong CP, Xiong M, Gao B (2011) Silver nanowires: from scalable synthesis to recyclable foldable electronics. Adv Mater 23(27):3052-3056

17. Hu L, Kim HS, Lee JY, Peumans P, Cui Y (2010) Scalable coating and properties of transparent, flexible, silver nanowire electrodes. ACS Nano 4(5):2955-2963

18. Jiang S, Sreethawong T, Lee SSC, Low MBJ, Win KY, Brzozowska AM, Teo SLM, Vancso GJ, Jańczewski D, Han MY (2015) Fabrication of copper nanowire films and their incorporation into polymer matrices for antibacterial and marine antifouling applications. Adv Mater Interfaces 2(3):1-8

19. Shahzadi K, Wu L, Ge X, Zhao F, Li H, Pang S, Jiang Y, Guan J, Mu X (2016) Preparation and characterization of bio-based hybrid film containing chitosan and silver nanowires. Carbohydr Polym 137:732-738

20. Zhang Z, Wang H, Li S, Li L, Li D (2015) Transparent and flexible cellulose nanofibers/silver nanowires/acrylic resin composite electrode. Compos Part A Appl Sci Manuf 76:309-315

21. Jiu J, Araki T, Wang J, Nogi $M$, Sugahara $T$, Nagao $S$, Koga $H$, Suganuma K, Nakazawa E, Hara M (2018) Facile synthesis of very-long silver nanowires for transparent electrodes. J Mater Chem A 2(14):6326-6330

22. Kumar B, Castro M, Feller J (2012) Poly(lactic acid)-multi-wall carbon nanotube conductive biopolymer nanocomposite vapour sensors. Sensors Actuators B Chem 161(1):621-628

23. Luo Z, Cai Z, Wang Y, Wang Y, Wang B (2016) In situ growth of silver nanowires on reduced graphene oxide sheets for transparent electrically conductive films. RSC Adv 6(43):37124-37129

24. Mi HY, Li Z, Turng LS, Sun Y, Gong S (2014) Silver nanowire/thermoplastic polyurethane elastomer nanocomposites: thermal, mechanical, and dielectric properties. Mater Des 56:398-404

25. Xu F, Zhu Y (2012) Highly conductive and stretchable silver nanowire conductors. Adv Mater 24(37):5117-5122

26. Hu W, Niu X, Li L, Yun S, Yu Z, Pei Q (2012) Intrinsically stretchable transparent electrodes based on silver-nanowire-crosslinkedpolyacrylate composites. Nanotechnology 23(34):344002

27. Zulfiqar AR, Asma R, Faiza A, Adil U (2016) Development and antibacterial performance of silver nanoparticles. Bull Mater Sci 39(2):391-396

28. Kazeminezhad I, Barnes AC, Holbrey JD, Seddon KR, Schwarzacher W (2007) Templated electrodeposition of silver nanowires in a nanoporous polycarbonate membrane from a nonaqueous ionic liquid electrolyte. Appl Phys A Mater Sci Process 86(3):373-375

29. Zeng XY, Zhang QK, Yu RM, Lu CZ (2010) A new transparent conductor: silver nanowire film buried at the surface of a transparent polymer. Adv Mater 22(40):4484-4488

30. Lee J, Lee $\mathrm{P}$, Lee HB, Hong S, Lee I, Yeo J, Lee SS, Kim TS, Lee D, Ko SH (2013) Room-temperature nanosoldering of a very long metal nanowire network by conducting-polymer-assisted joining for a flexible touch-panel application. Adv Funct Mater 23(34):4171-4176

31. Sun Y, Gates B, Mayers B, Xia Y (2002) Crystalline silver nanowires by soft solution processing. Nano Lett 2(2):165-168

32. Mazur M (2004) Electrochemically prepared silver nanoflakes and nanowires. Electrochem Commun 6(4):400-403

33. Sun XY, Xu FQ, Li ZM, Zhang WH (2005) Cyclic voltammetry for the fabrication of high dense silver nanowire arrays with the assistance of AAO template. Mater Chem Phys 90(1):69-72

34. Kim SH, Choi BS, Kang K, Choi YS, Yang SI (2007) Low temperature synthesis and growth mechanism of Ag nanowires. J Alloys Compd 433(1-2):261-264

35. Peng H, Yang A, Xiong JG (2013) Microwave-assisted synthesis of silver nanoparticles using bamboo hemicelluloses and glucose in an aqueous medium. Carbohydr Polym 91(1):348-355 
36. Pang H, Bai R, Shao Q, Gao Y, Li A, Tang Z (2015) A novel Ag catalyzation process using swelling impregnation method for electroless Ni deposition on Kevlar fiber. Appl Surf Sci 359:280-287

37. Vasylyev S, Damm C, Segets D, Hanisch M, Taccardi N, Wasserscheid P, Peukert W (2013) Synthesis of silver nanoparticles in melts of amphiphilic polyesters. Nanotechnology 24(11):115604

38. Lu Z, Meng M, Jiang Y, Xie J (2014) UV-assisted in situ synthesis of silver nanoparticles on silk fibers for antibacterial applications. Colloids Surf A Physicochem Eng Asp 447:1-7

39. Zulfiqar AR (2018) In situ synthesis and immobilization of nanosilver on knitted cellulose fabric. J Nat Fibers 15(2):183-190

40. Zulfiqar AR, Umaira B, Unsa N, Somayyah AM, Shahina R (2019) Chitosan mediated formation and impregnation of silver nanoparticles on viscose fabric in single bath for antibacterial performance. Fibers Polym 20(7):1360-1367

41. Montazer M, Komeily Nia Z (2015) Conductive nylon fabric through in situ synthesis of nano-silver: preparation and characterization. Mater Sci Eng C 56:341-347

42. Jiang SX, Qin WF, Guo RH, Zhang L (2010) Surface functionalization of nanostructured silver-coated polyester fabric by magnetron sputtering. Surf Coat Technol 204(21-22):3662-3667

43. Yu D, Kang G, Tian W, Lin L, Wang W (2015) Preparation of conductive silk fabric with antibacterial properties by electroless silver plating. Appl Surf Sci 357:1157-1162

44. Hebeish AA, Ramadan MA, Montaser AS, Farag AM (2014) Preparation, characterization and antibacterial activity of chitosang-poly acrylonitrile/silver nanocomposite. Int J Biol Macromol 68:178-184

45. Bélanger MC, Marois $Y$ (2001) Hemocompatibility, biocompatibility, inflammatory and in vivo studies of primary reference materials low-density polyethylene and polydimethylsiloxane: a review. J Biomed Mater Res 58(5):467-477

46. McDonald JC, Duffy DC, Anderson JR, Chiu DT, Wu H, Schueller OJ, Whitesides GM (2000) Fabrication of microfluidic systems in poly(dimethylsiloxane). Electrophoresis 21:27-40

47. Kuncová-Kallio PKJ (2006) PDMS and its suitability for analytical microfluidicdevices. In: Conference proceedigs of IEEE Engineering in Medice and Biology Society

48. Lamberti A, Marasso SL, Cocuzza M (2014) PDMS membranes with tunable gas permeability for microfluidic applications. RSC Adv 106:61415-61419

49. Marasso SL, Puliafito A, Mombello D, Benetto S, Primo L, Bussolino F, Pirri CF, Cocuzza M (2017) Optimized design and fabrication of a microfluidic platform to study single cells and multicellular aggregates in 3D. Microfluid Nanofluidics 21(2):29

50. Cui F, Chen W, Wu X, Guo Z, Liu W, Zhang W, Chen W (2017) Design and experiment of a PDMS-based PCR chip with reusable heater of optimized electrode. Microsyst Technol 23:3069-3079

51. Nguyen TPO, Tran BM, Lee NY (2016) Thermally robust and biomolecule-friendly room temperature bonding for the fabrication of elastomer-plastic hybrid microdevices. Lab Chip 16:3251-3259

52. Xia H, Mathew B, John T, Hegab H, Feng J (2013) Microfluidic based immunosensor for detection and purification of carbonylated proteins. Biomed Microdevices 15:519-530
53. Li X, Tian T (2018) Recent advances in an organ-on-a-chip: biomarker analysis and applications. Anal Methods 10:3122-3130

54. Bhatia SN, Ingber DE (2014) Microfluidic organs-on-chips. Nat Biotechnol 32(8):760-772

55. Caplin JD, Granados NG, James MR, Montazami R, Hashemi N (2015) Microfluidic organ-on-a-chip technology for advancement of drug development and toxicology. Adv Healthc Mater 4:1426-1450

56. Huh D, Hamilton GA, Ingber DE (2011) From 3D cell culture to organs-on-chips. Trends Cell Biol 21:745-754

57. Lee S, Jin SP, Kim YK, Sung GY, Chung JH, Sung JH (2017) Construction of 3D multicellular microfluidic chip for an in vitro skin model. Biomed Microdevices 19(2):22

58. Kae Sato, Kiichi Sato. Recent progress in the development of microfluidic vascular models Anal Sci, 34(7), 755-764, 2018

59. Bein A, Shin W, Jalili-Firoozinezhad S, Park MH, SontheimerPhelps A, Tovaglieri A, Chalkiadaki A, Kim HJ, Ingber DE (2018) Microfluidic organ-on-a-chip models of human intestine. Cell Mol Gastroenterol Hepatol 5(4):659-668

60. Potkay JA (2014) The promise of microfluidic artificial lungs. Lab Chip 14:4122-4138

61. Pasquardini L, Potrich C, Quaglio M, Lamberti A, Guastella S, Lunelli L, Cocuzza M, Vanzetti L, Pirri CF, Pederzolli C (2011) Solid phase DNA extraction on PDMS and direct amplification. Lab Chip 11(23):4029-4035

62. Potrich C, Vaghi V, Lunelli L, Pasquardini L, Santini GC, Ottone $C$, Quaglio M, Cocuzza M, Pirri CF, Ferracin M, Negrini M, Tiberio $P$, De Sanctis V, Bertorelli R, Pederzolli C (2014) OncomiR detection in circulating body fluids: a PDMS microdevice perspective. Lab Chip 14(20):4067-4075

63. Vaghi V, Potrich C, Pasquardini L, Lunelli L, Vanzetti L, Ebranati $E$, Lai A, Zehender G, Mombello D, Cocuzza M, Pirri CF, Pederzolli $C$ (2016) On-chip purification and detection of Hepatitis $C$ virus RNA from human plasma. Biophys Chem 208:54-61

64. Santini GC, Potrich C, Lunelli L, Vanzetti L, Marasso SL, Cocuzza M, Pirri FC, Pederzolli C (2017) miRNA purification with an optimized PDMS microdevice: toward the direct purification of low abundant circulating biomarkers. Biophys Chem 229:142-150

65. Makamba H, Kim JH, Lim K, Park N, Hahn JH (2003) Surface modification of poly (dimethylsiloxane) microchannels. Electrophoresis 24:3607-3619

66. Ibarlucea B, Fernández-Sánchez C, Demming S, Büttgenbach S, Llobera A (2011) Selective functionalisation of PDMS-based photonic lab on a chip for biosensing. Analyst 136:3496-3502

67. Zulfiqar Ali R, Asma R, Muhammad M, Sadia Z (2015) Development of antibacterial cellulosic fabric via clean impregnation of silver nanoparticles. J Clean Prod 101:377-386

Publisher's Note Springer Nature remains neutral with regard to jurisdictional claims in published maps and institutional affiliations. 